\title{
High performance silicon photonic devices based on practical metamaterials
}

\author{
Robert Halir ${ }^{1,2}$, Jose M. Luque-González ${ }^{1}$, Alejandro Sánchez-Postigo ${ }^{1}$, Alaine Herrero-Bermello ${ }^{3}$, David \\ González-Andrade ${ }^{3}$, Abdelfettah Hadij-El-Houati ${ }^{1}$, Jonas Leuermann ${ }^{2}$, Daniel Pereira-Martín ${ }^{1}$, Alejandro \\ Ortega-Moñux ${ }^{1}$, Jiří Čtyroký ${ }^{4}$, Gonzalo Wangüemert-Pérez ${ }^{1,2}$, Aitor V. Velasco ${ }^{3}$, Marina Sánchez- \\ Rodríguez $^{1}$, Jose de-Oliva-Rubio ${ }^{1}$, Jens H. Schmid ${ }^{5}$, Jordi Soler Penadés ${ }^{6}$, Milos Nedeljkovic ${ }^{6}$, \\ Goran Z. Mashanovich ${ }^{6}$, Pavel Cheben ${ }^{5}$, and Íñigo Molina-Fernández ${ }^{1,2}$ \\ ${ }^{1}$ Universidad de Málaga, Departamento de Ingeniería de Comunicaciones, ETSI Telecomunicación, Spain \\ ${ }^{2}$ Bionand Center for Nanomedicine and Biotechnology, Málaga, Spain \\ ${ }_{3}^{3}$ Instituto de Óptica, Consejo Superior de Investigaciones Científicas, Madrid, Spain \\ ${ }^{4} \mathrm{CAS}$, Institute of Photonics and Electronics, Chaberská 57, 18251 Prague, Czech Republic \\ ${ }^{5}$ National Research Council Canada, Ottawa, Ontario, Canada \\ ${ }^{6}$ Optoelectronics Research Centre, University of Southampton, Southampton, UK \\ rhalir@uma.es
}

(Invited)

\begin{abstract}
Subwavelength grating metamaterials are enabling a new generation of high-performance silicon photonic devices. Here we discuss the fundamental physics along with some of the latest advances in this rapidly expanding field.

Keywords: Si photonic and heterogeneous platform, Advanced Passive Devices, Optical filters, Optical polarization control devices, Novel Materials and Platform for Passive Devices
\end{abstract}

\section{INTRODUCTION}

Silicon photonics is attracting a lot of attention as a versatile platform for photonic integration, with applications ranging from high speed optical communications [1], to mid-infrared spectroscopy [2] and bio-sensing [3]. While CMOS compatibility is one of the advantages of this platform, it also restricts the materials, and hence refractive indexes, that can be employed. However, the high resolution afforded by modern lithography techniques enables the patterning of silicon waveguides at subwavelength scale, so that they behave as practical, all-dielectric metamaterials [4]. The ability to synthesize such metamaterials with a wide range of equivalent refractive indexes has opened new prospects for manipulating light in photonic chips. A wide variety of devices with unprecedented performance, including low loss fiberto-chip couplers, ultrabroadband multimode interference couplers, and narrowband Bragg filters, have been demonstrated [5], and are poised to become key building blocks in next generation photonic circuits. In this contribution we will briefly present the fundamentals of subwavelength materials and will then review some of the latest advances in this broad field.

\section{SubWaVelength Grating Metamaterials}

Figure 1(a) shows a silicon subwavelength grating (SWG) waveguide, with period $\Lambda$ and duty-cycle $a / \Lambda$. For periods matching or exceeding the Bragg condition $\Lambda=\lambda / n_{\text {eff }}$, where $n_{\text {eff }}$ is the mode effective index, the structure diffracts light backwards, or upwards and downwards [Fig. 1(b) center and top]. Below the Bragg threshold diffraction is suppressed and the structure behaves as a homogeneous waveguide composed of a metamaterial with a refractive index between that of silicon and the cladding [Fig. 1(b) bottom]. Considering the wide SWG structure shown in Figs. 1(c) and (d), it is clear that the Bloch-Floquet propagating along the $z$-direction [Fig. 1(c)] and the waveguide array mode propagating along the $x$-direction [Fig. 1(d)] experience difference effective indices, i.e. the metamaterial behaves like a uniaxial crystal with a permittivity tensor $\boldsymbol{n}^{2}=\operatorname{diag}\left(n_{x x}{ }^{2}, n_{x x}{ }^{2}, n_{z z}{ }^{2}\right)$ [4], [5]. The lithographically controlled duty-cycle of the structure governs the dielectric permittivity value, which can be tuned between that of silicon and the surrounding cladding, enabling the designer to choose the optimum metamaterial for each device. For a duty-cycle of $50 \%$ and $\Lambda=200 \mathrm{~nm}$, we find $n_{x x} \approx 2.79$ and $n_{z z} \approx 1.94$ at $\lambda=1.55 \mu \mathrm{m}$. The refractive index dispersion can be controlled using an appropriate period: small, deepsubwavelength periods yield reduced dispersion, whereas periods closer to the bandgap produce higher dispersion.

\section{High Performance Subwavelength Devices}

The properties of SWG waveguides can be exploited to enhance the performance of a wide variety of silicon photonic devices. An extremely important application is fiber-to-chip coupling, where SWG are used to gradually deconfine the

Funding: Ministerio de Economía y Competitividad, Programa Estatal de Investigación Orientada a los Retos de la Sociedad (cofinanciado FEDER) - TEC2016-80718-R, TEC2015-71127-C2-1-R (FPI BES-2016-077798) and IJCI-2016-30484; Community of Madrid - S2018/NMT-4326, Marie Sklodowska-Curie -734331, Universidad de Málaga, Czech Science Foundation - 1900062S. 
(a)

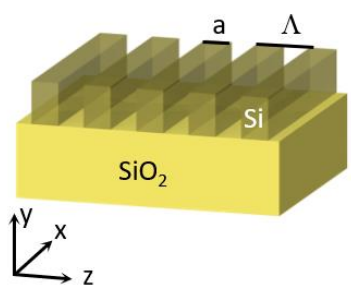

(c)

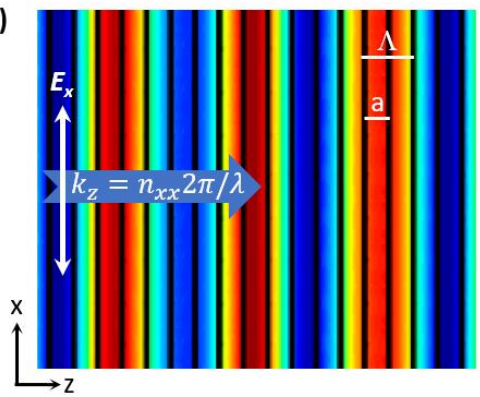

(b)

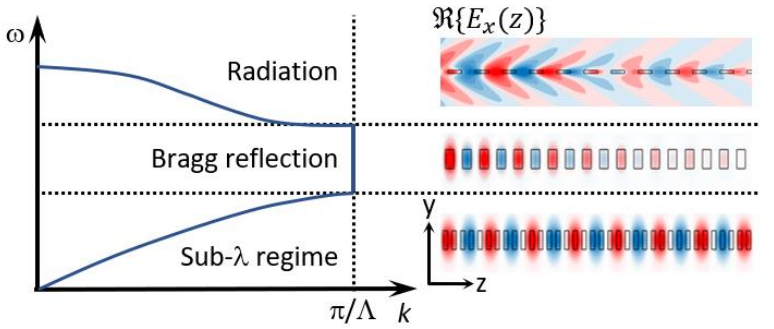

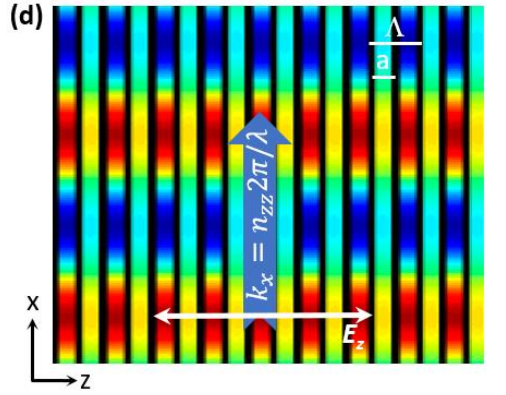

Fig. 1. Fundamentals of SWG waveguides. (a) A fully etched periodic silicon photonic waveguide. (b) When the period $(\Lambda)$ of the structure is smaller than the wavelength of light propagating through it, it behaves like a homogenous metamaterial. The homogenous metamaterial is anisotropic, as light propagating along the $z$-direction (panel c) and $x$-direction (panel d) experiences different refractive indices.

waveguide mode, offering broadband and polarization insensitive coupling [6]. Another example is the multimodeinterference coupler shown in Fig. 2(a), which acts as broadband $2 \times 2$ coupler, splitting the input power equally between its two outputs with a $90^{\circ}$ phase shift. The device exhibits a bandwidth in excess of $300 \mathrm{~nm}$ at telecom wavelengths, effectively tripling the bandwidth of conventional devices. This is achieved by exploiting the anisotropy of the SWG, which significantly shortens the device and reduces its wavelength dependence [7]. Combined with appropriate phase shifters and Y-junctions, this device also enables extremely broadband mode (de)multiplexing [8]. The anisotropy of a judiciously designed SWG, oriented parallel to the direction of propagation, has furthermore been demonstrated to reduce coupling between adjacent waveguides [9].

The reduced index of an SWG waveguide yields an expanded mode field, enhancing its interaction with the material surrounding the waveguide. This can be exploited to achieve a very weak, yet highly controlled interaction with loading segments in the waveguide vicinity - see Fig. 2(b). By separating these segments by the Bragg period, very narrow filters with high extinction ratios can be designed [10]. Using the additional degree of freedom afforded by a shift, $\xi$, between the loading segments this approach has enabled the demonstration of filter bandwidths as narrow as $200 \mathrm{pm}$ with $30 \mathrm{~dB}$ extinction [11]. The enhanced modal interaction can also be exploited to improve the sensitivity of photonic biosensors [12], which through careful design of the complete sensing system will result in better detection limits [13].

The mid-infrared wavelength region is of great interest for applications in environmental monitoring and security, as many chemical substance exhibit highly specific absorption peaks in this wavelength range. Since silicon dioxide absorbs strongly at wavelengths beyond $4 \mu \mathrm{m}$, silicon-on-insulator waveguides have to be suspended to operate in most of the mid-infrared. Using an SWG cladding, as shown in Fig. 2(c), enables the penetration of hydrofluoric acid to remove the silicon dioxide layer, requiring only one etch step. Using this approach, we have demonstrated waveguides with subdecibel per centimeter losses near 3.8 $\mu \mathrm{m}$ wavelength, $3 \mathrm{~dB} / \mathrm{cm}$ losses near $7.7 \mu \mathrm{m}$ [14], and suspended MMIs [15].

A further degree of freedom of SWG waveguides which has scarcely been exploited so far is the inclination (tilt) of the segments - see Fig. 2(d). We have recently shown that tilting affects mainly the TE polarized modes, with virtually no effect on the TM polarized modes [16]. As shown in the inset, the phase front of the TE modes tilts strongly when the segments are tilted, whereas the phase front of the TM modes is much less distorted. This enables direct engineering of the waveguide birefringence, with the added advantage that the effective index can be tuned without altering the dutycycle. This results in structure with constant feature size which facilitates accurate fabrication. This approach is especially useful for designing devices that manipulate polarization. We have proposed an MMI with tilted SWG segments that acts polarization splitter covering a $130 \mathrm{~nm}$ bandwidth using a constant minimum feature size of $110 \mathrm{~nm}$ [17], [18]. Using a "mixed" anisotropy approach a polarization splitter covering more than 200nm with a minimum feature size of $65 \mathrm{~nm}$ has been recently demonstrated [19].

\section{CONClusions}

The flexibility afforded by subwavelength metamaterials, combined with the continuous advances in silicon lithography is driving the development of a new generation of high-performance devices. As engineering of not only the local refractive index, but also of dispersion and anisotropy is increasingly well understood and used advantageously by research groups and industry around the globe, subwavelength structure as becoming an essential tool in silicon photonics. 
(a)

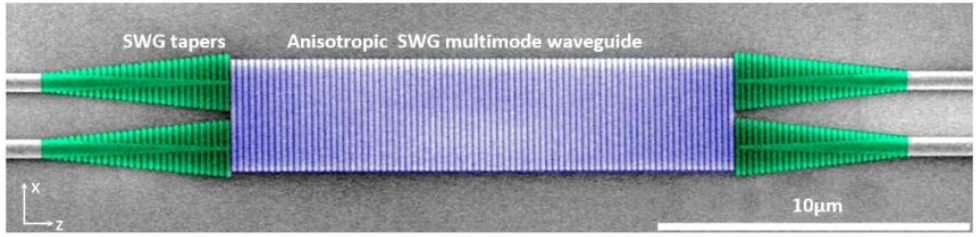

(b)

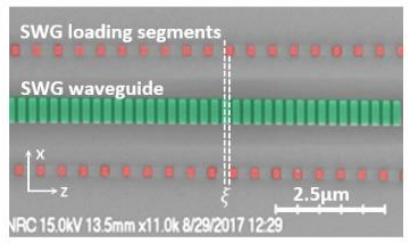

(c)

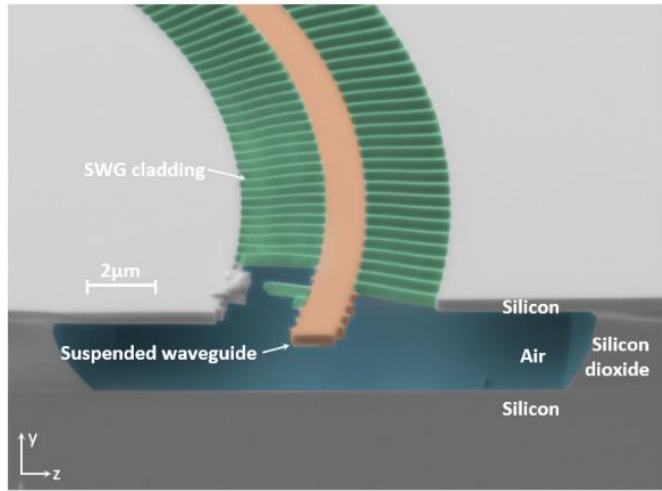

(d)

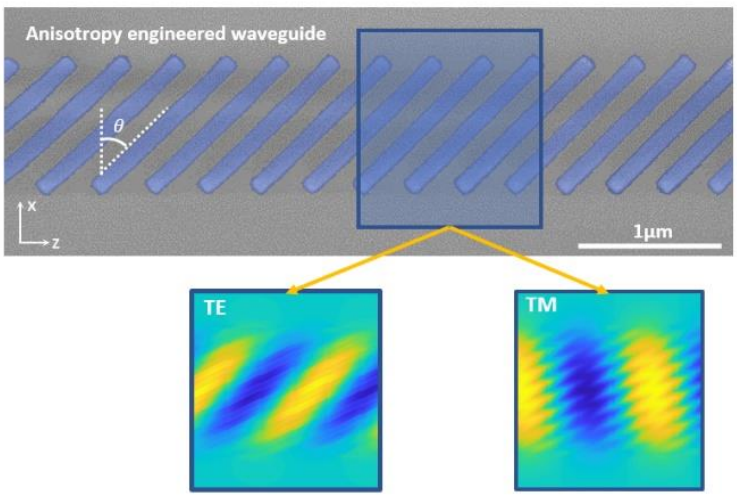

Fig. 2. Applications of subwavelength gratings. (a) A beam splitter with an experimental bandwidth in excess of 300nm, based on multimode interference in an anisotropic metamaterial [7]. (b) A subwavelength waveguide loaded with subwavelength Bragg segments enables measured bandwidths down to $150 \mathrm{pm}$ [10], [11]. (c) Waveguides with subwavelength cladding allow for suspended waveguide which exhibit low losses in the mid-infrared [14], [15]. (d) Tilting of subwavelength segments provides fine control over waveguide birefringence; the insets show the field propagation of the TE and TM modes [16]. The scanning electron microscope pictures have been colored to highlight different parts of the devices.

\section{REFERENCES}

[1] C. Doerr and L. Chen, "Silicon Photonics in Optical Coherent Systems," Proc. IEEE, vol. 106, no. 12, pp. 2291-2301, Dec. 2018.

[2] J.-M. Fedeli and S. Nicoletti, "Mid-Infrared (Mid-IR) Silicon-Based Photonics," Proc. IEEE, vol. 106, no. 12, pp. 23022312, Dec. 2018

[3] E. Luan, H. Shoman, D. Ratner, K. Cheung, and L. Chrostowski, "Silicon Photonic Biosensors Using Label-Free Detection," Sensors, vol. 18, no. 10, p. 3519, Oct. 2018.

[4] P. Cheben, R. Halir, J. H. Schmid, H. A. Atwater, and D. R. Smith, "Subwavelength integrated photonics," Nature, vol. 560, no. 7720, pp. 565-572, Aug. 2018.

[5] R. Halir et al., "Subwavelength-Grating Metamaterial Structures for Silicon Photonic Devices," Proc. IEEE, vol. 106, no. 12, pp. 2144-2157, Dec. 2018.

[6] P. Cheben et al., "Broadband polarization independent nanophotonic coupler for silicon waveguides with ultra-high efficiency," Opt. Express, vol. 23, no. 17, pp. 22553-22563, 2015.

[7] R. Halir et al., "Ultra-broadband nanophotonic beamsplitter using an anisotropic sub-wavelength metamaterial," Laser Photon. Rev., vol. 10, no. 6, pp. 1039-1046, Nov. 2016.

[8] D. González-Andrade et al., "Ultra-Broadband Mode Converter and Multiplexer Based on Sub-Wavelength Structures," IEEE Photonics J., vol. 10, no. 2, pp. 1-10, Apr. 2018.

[9] S. Jahani et al., "Controlling evanescent waves on-chip using all-dielectric metamaterials for dense photonic integration," Nat. Commun., vol. 9, p. 1893, 2018.

[10] J. Čtyroký et al., "Design of narrowband Bragg spectral filters in subwavelength grating metamaterial waveguides," Opt. Express, vol. 26, no. 1, pp. 179-194, Jan. 2018.

[11] P. Cheben et al., "Bragg filter bandwidth engineering in subwavelength grating metamaterial waveguides," Opt. Lett., vol. 44, no. 4, pp. 1043-1046, Feb. 2019.

[12] J. G. Wangüemert-Pérez et al., "Subwavelength structures for silicon photonics biosensing," Opt. Laser Technol., vol. 109, pp. 437-448, Jan. 2019.

[13] Í. Molina-Fernández, J. Leuermann, A. Ortega-Moñux, J. G. Wangüemert-Pérez, and R. Halir, "Fundamental limit of detection of photonic biosensors with coherent phase read-out," Opt. Express, 2019.

[14] J. S. Penadés et al., "Suspended silicon waveguides for long-wave infrared wavelengths," Opt. Lett., vol. 43, no. 3, pp. 795798, 2018.

[15] A. Sánchez-Postigo et al., "Mid-infrared suspended waveguide platform and building blocks," IET Optoelectron., Oct. 2018.

[16] J. M. Luque-González et al., "Tilted subwavelength gratings: controlling anisotropy in metamaterial nanophotonic waveguides," Opt. Lett., vol. 43, no. 19, pp. 4691-4694, Oct. 2018.

[17] A. Herrero-Bermello, J. M. Luque-González, A. V Velasco, A. Ortega Moñux, P. Cheben, and R. Halir, "High efficiency polarization beam splitter based on anisotropy-engineered MMI," in Fiber Lasers and Glass Photonics: Materials through Applications, 2018, vol. 10683, p. 33.

[18] R. Halir et al., "Designing Anisotropy with Waveguide Subwavelength Structures," in 2018 20th International Conference on Transparent Optical Networks (ICTON), 2018, p. Mo.D5.4.

[19] H. Xu, D. Dai, and Y. Shi, "Ultra-Broadband and Ultra-Compact On-Chip Silicon Polarization Beam Splitter by Using Hetero-Anisotropic Metamaterials,” Laser Photon. Rev., p. 1800349, Feb. 2019. 